Brézins, Saint-Étienne-de-Saint-Geoirs - Le Grand

Plan, les Ardelières, Mas de Noyeraie, Grand

Vulpin, Petit Vulpin

Opération préventive de diagnostic (2013)

\title{
Fabien Isnard
}

\section{OpenEdition}

\section{Journals}

Édition électronique

URL : https://journals.openedition.org/adlfi/15059

ISSN : 2114-0502

Éditeur

Ministère de la Culture

Référence électronique

Fabien Isnard, « Brézins, Saint-Étienne-de-Saint-Geoirs - Le Grand Plan, les Ardelières, Mas de Noyeraie, Grand Vulpin, Petit Vulpin » [notice archéologique], ADLFI. Archéologie de la France Informations [En ligne], Auvergne-Rhône-Alpes, mis en ligne le 30 juillet 2015, consulté le 28 juillet 2021. URL : http://journals.openedition.org/adlfi/15059

Ce document a été généré automatiquement le 28 juillet 2021.

(c) ministère de la Culture et de la Communication, CNRS 


\section{Brézins, Saint-Étienne-de-Saint- Geoirs - Le Grand Plan, les Ardelières, Mas de Noyeraie, Grand Vulpin, Petit Vulpin}

Opération préventive de diagnostic (2013)

\section{Fabien Isnard}

\section{NOTE DE L'ÉDITEUR}

Organisme porteur de l'opération : Inrap

Cette série de sondages-diagnostics situés en zone de saisine archéologique au milieu de la vallée de la Bièvre, sur la commune de Brézins, s'est avérée largement positive. En effet, une occupation dense et multi-périodes a été mise en évidence à l'intérieur de l'emprise des deux bassins écrêteurs de crues à l'origine de la prescription. Quatorze des trente-sept sondages réalisés ont révélé des structures archéologiques. Un tiers des sondages sont positifs dans le bassin sud, et la moitié des sondages sont positifs dans le bassin nord. Ces occupations et aménagements ont été associés à quatre périodes distinctes, allant du Néolithique moyen à la période contemporaine. Au niveau du bassin sud, une occupation protohistorique, voire plus ancienne et liée à l'exploitation d'un marécage, a été mise en évidence. Une fosse à incinération secondaire datée de l'âge du Bronze final a été repérée et le sommet de son contenu analysé. Celui-ci a révélé du matériel d'une extrême finesse associé à cette structure. Selon la littérature archéologique concernant ce mode d'inhumation durant ces périodes, cette urne ne devrait pas se trouver isolée. Dans l'emprise du bassin nord, l'occupation néolithique et protohistorique se poursuit sous la forme de structures fossoyées et de foyers ou fours en batterie. 
2 Mais la découverte majeure de ce diagnostic concerne surtout la mise au jour d'un tronçon de la voie romaine qui reliait l'Italie à Vienne en passant par cette vallée. Cette voie antique de circulation connue par les textes n'avait pas encore été localisée précisément dans ce secteur. Il s'agit donc d'une découverte importante en ce qui concerne la connaissance et le calage de cet axe de circulation.

3 La plaine de la Bièvre, et plus particulièrement les secteurs de la Côte-Saint-André, Brézins et Saint-Étienne-de-Saint-Geoirs ont fait l'objet de nombreuses découvertes archéologiques depuis le $\mathrm{xIX}^{\mathrm{e}} \mathrm{s}$. Durant plusieurs campagnes de prospections en 2010 et 2011, doublées d'une étude d'archives et de photos aériennes, Denis Gonin a pu mettre en évidence la potentialité de nombreux sites allant de la période néolithique à l'époque médiévale. Des opérations de diagnostics menées par l'Inrap ont confirmé cette densité d'occupation de la zone, notamment dans toute la Zac du Rival (Bleu 2009 et 2010) où plusieurs fouilles ont été réalisées (Pequignot 2011; Bocquet 2013).

Pour les périodes préhistorique et néolithique, les découvertes isolées de pièces de silex taillées autour de la commune de Brézins sont assez rares, mais néanmoins présentes.

5 Ces découvertes se densifient pour l'âge du Bronze, avec la présence notamment dans le secteur du Rival, distant de quelques kilomètres, du fameux char processionnel en bronze retrouvé dans un tumulus à la fin du $\mathrm{xIX}^{\mathrm{e}} \mathrm{s}$.

6 D'autres découvertes de types enclos ou cercles de pierres concernant l'âge des métaux ont été mises en évidence dans l'axe est-ouest le long du cours du Rival, dans une bande d'occupation correspondant au fond du vallon.

7 Pour l'époque romaine, le village de Brézins semble être installé sur un site, au vu de la présence de nombreux murs et dallages inventoriés. Un aqueduc antique encore en fonctionnement est même répertorié sur la commune, et il alimente encore en eau une entreprise locale. Cette villa, hameau ou relais routier, était sans doute en relation avec la grande voie romaine qui reliait Vienne à l'Italie via Grenoble. Cet axe principal de circulation, qui traversait la plaine de la Bièvre, était déjà répertorié sur la Table de Peutinger, sans être précisément localisé. Cependant, de nombreux sites ont été localisés le long d'un axe est-ouest passant par Brézins et coupant notre emprise de diagnostic, notamment au lieu-dit du Champ Canel, où un dépôt monétaire du BasEmpire a été découvert au mas de la Vie de Lariot en 1979 par le propriétaire du terrain. Ce dépôt se trouve actuellement au Musée dauphinois à Grenoble (SRA, carte archéologique EA 375 ; Gonin 2009).

8 L'époque médiévale est tout aussi riche en vestiges, comme l'attestent de nombreux édifices sur la commune de Brézins, ainsi que l'occupation dense révélée lors de la fouille de la Zac du Rival (Bocquet 2013). Ainsi, outre l'occupation dense de ces secteurs entre le Néolithique et l'âge du Bronze, une fosse à incinération secondaire a été découverte dans le sondage 10. De nombreux fragments de céramique fine et noire décorés à l'étain ont été récoltés en surface, ainsi qu'un manche de rasoir en bronze comme il en existe sur le site du lac du Bourget à l'âge du Bronze final.

9 En ce qui concerne le tronçon de voie romaine découvert lors de ce diagnostic, les caractéristiques de cette chaussée, reconnue sur une longueur de plus de $90 \mathrm{~m}$, permettent d'avancer comme hypothèse sérieuse qu'il s'agit là d'un tronçon de voie antique implanté dans la vallée de la Bièvre. Il doit probablement être rattaché à un axe important, appartenant au groupe des voies alpines, qui permettait de rallier Vienne depuis l'Italie par le col du Mont Genèvre. À ce jour, la localisation de la voie n'est pas 
encore précisément établie dans le secteur de Brézins. Il est par conséquent vraisemblable que les vestiges dégagés durant le diagnostic correspondent à un nouveau tronçon de cette voie antique, à mi-chemin entre Izeaux et Tourdan. L'établissement de cette voie dans la vallée de la Bièvre respecte les normes mises en place par les Romains pour pérenniser les axes de circulation. On remarquera que cet axe garde un tracé en ligne droite autant que possible, mais en utilisant malgré tout le fond de la vallée. Par contre, il évite soigneusement les zones marécageuses, comme c'est le cas ici, en rive gauche du Rival. La réalisation de la route est très soignée afin que l'on puisse y circuler en toutes saisons. Son axe passe par Brézins, en son centre, en arrivant plein ouest. Du point de vue des dimensions et de l'épaisseur conservée, la voie mise au jour à Brézins entre tout à fait dans les gabarits de voies régionales retrouvées dans le secteur. Ainsi, la voie fouillée dans la plaine de Vaise, au nord-ouest de Lyon, présente une épaisseur qui n'excède pas une quinzaine de centimètres (Chastel 1995, p. 44-53). La voie se décompose en deux entités : la chaussée proprement dite et un bascôté, pour une largeur totale de 11,30 m. Sur le site de Lachar à Varces (Isère), la voie a pu être repérée sur une largeur comprise entre 6 et $8 \mathrm{~m}$, pour une épaisseur d'environ $20 \mathrm{~cm}$ (Guyon, Plassot 1996, p. 131-133 et 137). Les travaux de E. Ferber à Allan (Drôme) ont permis de découvrir un tronçon qui matérialise le decumanus DD XXXV du cadastre B d'Orange (Ferber 1996, p. 3 et 51). La voie d'une largeur approximative de $4 \mathrm{~m}$ (épaisseur : $30 \mathrm{~cm}$ ) est bordée au nord par un fossé profond d'environ $60 \mathrm{~cm}$.

Enfin, l'exemple le plus proche, puisqu'il s'agirait de la même voie, est celui de Moirans (Isère), où Franck Gabayet a dégagé un tronçon de voie de 6 à $7 \mathrm{~m}$ de large conservé sur 0,4 à $0,5 \mathrm{~m}$ de profondeur. Celle-ci était constituée d'une succession de couches sensiblement similaires à notre voie et bordée d'un seul fossé (Gabayet 1997, p. 5-9). 


\section{INDEX}

chronologie https://ark.frantiq.fr/ark:/26678/pcrtwpx5MU2hlw, https://ark.frantiq.fr/ark:/ 26678/pcrtH8P95EucZz, https://ark.frantiq.fr/ark:/26678/pcrtHlenwSnkDM, https:// ark.frantiq.fr/ark:/26678/pcrtGTWPtWn8qu, https://ark.frantiq.fr/ark:/26678/pcrtxT02uJOogm, https://ark.frantiq.fr/ark:/26678/pcrtof7EHNsS2e, https://ark.frantiq.fr/ark:/26678/ pcrtZTmusVUU24 nature https://ark.frantiq.fr/ark:/26678/pcrtWWQS75V5Bc lieux https://ark.frantiq.fr/ark:/26678/pcrtSEeAipsBlD, https://ark.frantiq.fr/ark:/26678/ crtB8WDyqd6u9, https://ark.frantiq.fr/ark:/26678/pcrtjNthkbl8NF, https://ark.frantiq.fr/ark:/ 26678/pcrtrN4UvuR9vH, https://ark.frantiq.fr/ark:/26678/crtjbLtlofHA8

Année de l'opération : 2013

sujets https://ark.frantiq.fr/ark:/26678/pcrtms2OAv82PY, https://ark.frantiq.fr/ark:/26678/ pcrtJUwAaZ7Nz9, https://ark.frantiq.fr/ark:/26678/pcrtIqEHwJLuq0, https://ark.frantiq.fr/ark:/ 26678/pcrtjclbYvph1S, https://ark.frantiq.fr/ark:/26678/pcrt1eKrSANesO, https:// ark.frantiq.fr/ark:/26678/pcrtSVdwKcS3MI, https://ark.frantiq.fr/ark:/26678/pcrtj3ZcwkArSR, https://ark.frantiq.fr/ark:/26678/pcrt4N9jrETRto, https://ark.frantiq.fr/ark:/26678/ pcrtIzSUofuocv, https://ark.frantiq.fr/ark:/26678/pcrtKJVpuP3AET, https://ark.frantiq.fr/ark:/ 26678/pcrtDlzbGxWvTo, https://ark.frantiq.fr/ark:/26678/pcrtbptj4SOA1W, https:// ark.frantiq.fr/ark:/26678/pcrtLEIWWX4Z30, https://ark.frantiq.fr/ark:/26678/ pcrt1DMOWvDF4j, https://ark.frantiq.fr/ark:/26678/pcrtQKxf8QNzai

\section{AUTEURS}

\section{FABIEN ISNARD}

Inrap 